\title{
Investigating characteristics of liquid hydrocarbon spraying by a steam jet
}

\author{
Igor Anufriev, Evgeniy Kopyev, and Evgeniy Shadrin* \\ Kutateladze Institute of Thermophysics SB RAS, Novosibirsk, Russia
}

\begin{abstract}
Using the shadow photography method, the dispersed composition of a gas-droplet flow has been investigated at diesel fuel spraying by a superheated steam jet. The distributions of droplet sizes have been obtained in a wide range of parameters (steam and fuel consumption). Dependencies of droplets size on steam consumption have been found. The optimum values of steam and fuel consumption, at which the smallest prevailing droplet size (below $30 \mu \mathrm{m}$ ) is reached, have been found.
\end{abstract}

Jet streams created by injectors are currently used in various fields of science and technology: cooling and fire extinguishing systems, medical inhalers, internal combustion engines, boiler equipment and many others. To improve the operation of devices with spraying nozzles, a detailed study of characteristics of the generated gas-droplet flow is required. Obtaining information on the velocity distribution and droplet sizes in the investigated jet stream allows justifying scientifically the selection of optimal regime parameters of the device. In particular, it is important when designing effective burners, used for utilization of substandard hydrocarbon fuels and industrial waste. Studies of the combustion of such liquid hydrocarbons, carried out by the authors earlier [1-3], have shown that the process of their ignition and combustion is greatly intensified when a superheated steam jet is fed into the combustion zone. The environmental characteristics of the process are improved as well. However, the use of evaporative-type burner devices does not allow achieving high productivity (power).

In this paper, a promising method of spraying a liquid hydrocarbon fuel with a steam jet [4] is experimentally studied. A distinctive feature of this method is that the fuel and spraying medium (carrier phase - steam), are not pre-mixed: the steam is supplied from the nozzle in the form of a jet, on which liquid fuel flows, thereby forming a fine gas-droplet flow. In practice this is an important advantage because of no contact between the fuel and the nozzle, and, hence, no coking of its surfaces and subsequent failures in the burner operation.

Using the shadow photography method [5] the dispersed composition of the gas-droplet flow was investigated at diesel fuel spraying by a superheated steam jet. The experimental setup is shown in Figure 1. This method is based on registering a shadow photograph of an object having a refractive index, different from ambient medium. At that, behind the investigated object (relative to the camera) there is a diffuse light source with a uniform

*Corresponding author: evgen_zavita@mail.ru 
spatial distribution of intensity. The focusing plane of the camera lens is close to the object of investigation to obtain the greatest sharpness of the shadow photo. Digital analysis of the shadow image allows identifying the boundary and position of the object. This method allows measuring the droplet sizes within the range from 10 to $1000 \mu \mathrm{m}$.

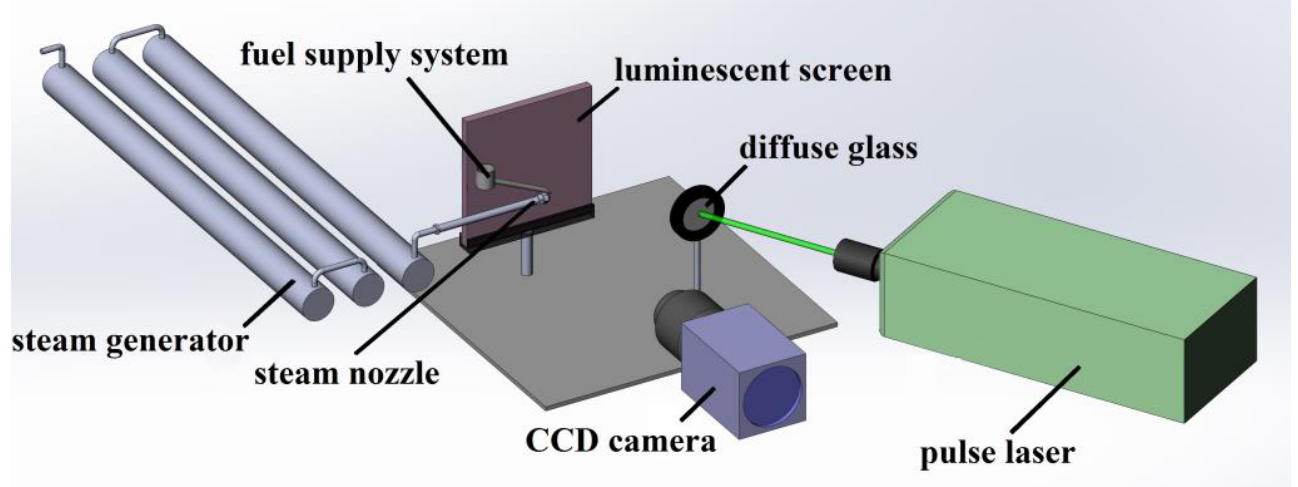

Fig.1. Scheme of experimental setup for studying characteristics of liquid fuel sprayed with a steam jet.

In the experiments, the CCD camera Videoscan 4021 (with a resolution of $2048 \times 2048$ pixels, a shooting frequency up to $1.25 \mathrm{~Hz}$, and an exposure time of $28 \mathrm{~ms}$ ) and the Tamron SP AF macro lens with a focal length of $180 \mathrm{~mm}$ were used to reduce the size of the measuring area to $10 \times 10 \mathrm{~mm}$ with good spatial resolution (magnification 1.5:1). A background screen with a rhodamine-based luminescent coating preliminarily illuminated by a defocused beam of a Nd:YAG QuantelEVG pulse laser (wavelength $532 \mathrm{~nm}$, pulse energy up to $145 \mathrm{~mJ}$, and pulse duration $10 \mathrm{~ns}$ ) was used as a light source. A threshold light filter $(560 \mathrm{~nm})$ whose bandwidth corresponds to the wavelength of light re-emitted by rhodamine was used to increase the shadow photo contrast. A coordinate-moving device with a positioning accuracy of $0.1 \mathrm{~mm}$ was used to move the camera along the axis of the nozzle that enabled examining of the flow at different distances from the nozzle.

The measurements were carried out at different distances from the nozzle and at different operating parameters: the steam flowrate was varied within $100-600 \mathrm{~g} / \mathrm{h}$, and the fuel consumption ranged to $300-600 \mathrm{~g} / \mathrm{h}$. The temperature of the superheated steam at the outlet from the steam generator was $150-550^{\circ} \mathrm{C}$, and a pressure inside the steam generator was up to $10 \mathrm{~atm}$.

For digital processing of the obtained shadow images the "Bubbles Identification" algorithm, implemented in the ActualFlow software [6], was used. It includes high-pass filtering algorithm to identify the boundaries of images of registered objects, algorithm for binarization by a threshold value, and algorithm for determining the position and the diameter of spherical droplets. Figure 2 shows the image processed using the specified algorithm.

Image processing has resulted in the data on the dispersed composition of fuel droplets under various spraying regimes. Figure 3 shows the characteristic dependences of the droplet size on steam flowrate and distance from the nozzle. The analysis of the results shows (Fig. 3-a) that the droplets size depends on steam flowrate: the share of small droplets in the flow $(10-20 \mu \mathrm{m})$ increases with the steam consumption (vapor velocity at the outlet from the nozzle). At the same time, the droplets size is almost independent of the distance from the nozzle (Fig. 3-b), which indicates the efficiency of fuel spraying by the proposed method. There is a slight decrease in the size of the droplets as they move away 
from the nozzle, which, apparently, is associated with evaporation. At steam consumption of $600 \mathrm{~g} / \mathrm{h}$ and fuel consumption of $300 \mathrm{~g} / \mathrm{h}$, the prevailing droplets size in the flow is 10-30 $\mu \mathrm{m}$. An increase in fuel consumption or a decrease in steam consumption led to an increase in the characteristic droplets size.

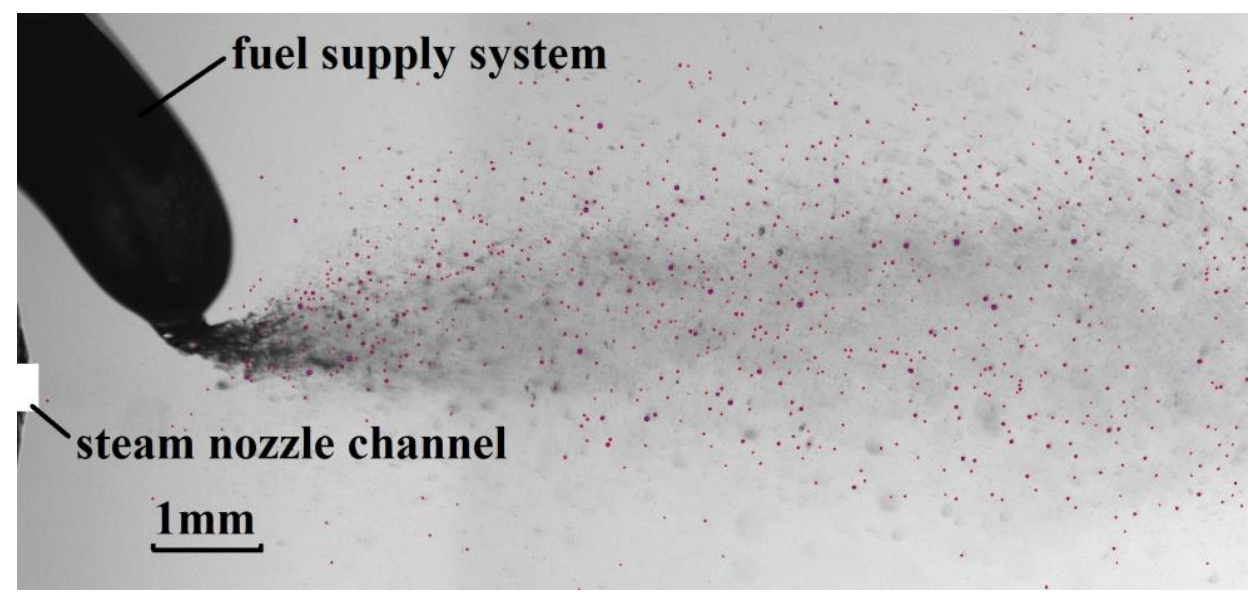

Fig.2. Result of processing of the shadow image of the fuel, sprayed with a steam jet in the air (the identified particles are indicated by marks).

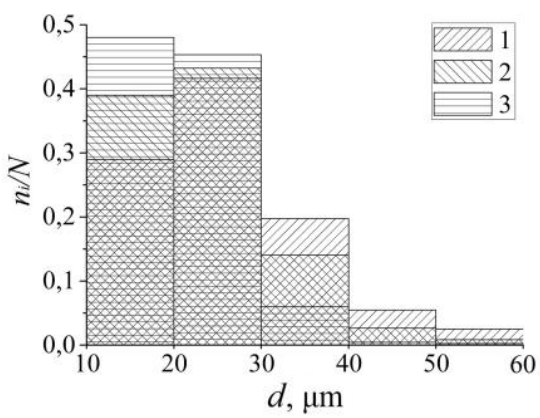

(a)

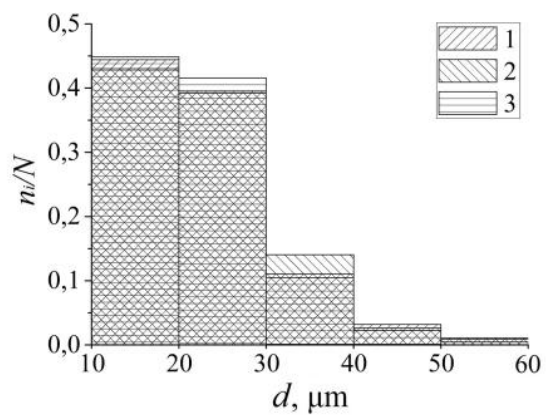

(b)

Fig.3. Dispersed composition of fuel droplets, formed by spraying diesel fuel with a superheated steam jet in the atmosphere: (a) for different steam consumption $(1-100 \mathrm{~g} / \mathrm{h}, 2-300 \mathrm{~g} / \mathrm{h}, 3-600$ $\mathrm{g} / \mathrm{h}$ ), with a fuel consumption of $300 \mathrm{~g} / \mathrm{h}$, at a distance of $10 \mathrm{~mm}$ from the nozzle; (b) at different distances from the nozzle $(1-0-10 \mathrm{~mm}, 2-10-20 \mathrm{~mm}, 3-20-30 \mathrm{~mm})$, at a steam consumption of $300 \mathrm{~g} / \mathrm{h}$ and a fuel consumption of $300 \mathrm{~g} / \mathrm{h}$. ( $n_{i}$ is the number of droplets with dimensions from a certain range, and $N$ is the total number of droplets identified by the algorithm, $d$ - is the size of droplet).

As a result, the characteristics of the liquid hydrocarbon fuel spraying by a superheated steam jet have been studied using the method of shadow photography. The optimal values for steam and fuel consumption have been found, which are 600 and $300 \mathrm{~g} / \mathrm{h}$ for steam and fuel, respectively. At that, the smallest prevailing droplets size (below $30 \mu \mathrm{m}$ ) in the flow is achieved. This dispersion result ensures efficient fuel combustion. At the same time, the effect of superheated steam temperature on the characteristics of spraying is not noticeable.

The research was carried out with the financial support of the Russian Science Foundation (project No. 17-79-20209) 


\section{References}

1. S.V. Alekseenko, I.S. Anufriev, M.S. Vigriyanov, E.P. Kopyev, O.V. Sharypov, Combustion, Explosion, and Shock Waves, 52, 286 (2016)

2. S.V. Alekseenko, I.S. Anufriev, M.S. Vigriyanov, V.M. Dulin, E.P. Kopyev, O.V. Sharypov, Thermophysics and Aeromechanics, 21, 393 (2014)

3. I.S. Anufriev, E.P. Kopyev, E.L. Loboda, Proc. SPIE, 9292, (2014)

4. M.S. Vigriyanov, S.V. Alekseenko, I.S. Anufriev, O.V. Sharypov, RF Patent No. 2579298 (2016) [in Russian]

5. G.V. Kuznetsov, P.A. Strizhak, R.S. Volkov, O.V. Vysokomornaya, Int. J. Therm. Sci., 108, 218 (2016)

6. User's Guide: Identify images of circular objects by delineating borders on images obtained by shadow photography and PFBI methods, (2012) [in Russian] 